\title{
Os Sistemas de workflow em arquivística: a identificação dos modelos e a análise das ferramentas
}

\section{Sérgio Renato Lampert}

Arquivista do Centro Universitário Franciscano. Epecializado do Curso de Gestão de Arquivos da UAB/UFSM.

Daniel Flores

Docente do Curso de Arquivologia da UFSM. Doutor em Documentação pela Universidade de Salamanca Espanha. Líder do Grupo de Pesquisa CNPq - Gestão Eletrônica de Documentos (GED).

Apresenta a tecnologia de workflow sob a ótica da arquivística, apontando os sistemas de workflow, bem como a identificação dos modelos e a análise das ferramentas. Com base nos critérios deste estudo, foi possível identificar uma gama de ferramentas que promovem funções arquivísticas. A análise deu-se apenas nas ferramentas identificadas como sendo Sistemas de Gestão Documental (DMS), as quais são: Agorum Core, Alfresco, ArchivistaBox, KnowledgeTree, Maarch e Owl Intranet.

Palavras-chave: Arquivística; Gestão Eletrônica de Documentos; Tecnologia da Informação; Workflow.

\section{Workflow systems in archival: identification of models and analysis tools}

Present Workflow technology from perspective of archival, indicating the workflow systems, as well as identification of models and analysis tools. Based on the criteria from study was to identify possible of products a tools that promote archival functions. The analysis gave only tools those identified as Document Management System (DMS), which are: Agorum Core, Alfresco, ArchivistaBox, KnowledgeTree, Maarch e Owl Intranet.

Keywords: Archival; Document Management; Information Technology; Workflow. 


\section{Recebido em 13.03.2010 Aceito em 18.10.2010}

\section{Introdução}

A Gestão Eletrônica de Documentos (GED) está em crescente produção científica. Estudos sobre o tema ganham cada vez mais espaço em congressos e eventos de sua área de abrangência. Contudo, a GED é uma área do conhecimento muito ampla e possui diversas tecnologias que permitem 0 Gerenciamento da Informação em meio eletrônico e, dentre essas, destaca-se o workflow.

Assim sendo, por ser uma tecnologia presente nas mais diversas instituições que trabalham com processos e fluxos de trabalho, a tecnologia de workflow passa a ter relevância no que diz respeito aos procedimentos executados pelos profissionais que atuam com documentos digitais.

Embora o tema pareça específico, ele é muito abrangente, uma vez que os Sistemas de workflow possuem diversos modelos e inúmeras ferramentas capazes de gerir um trâmite documental e/ou um fluxo de trabalho. Desse modo, a abordagem dada ao estudo dos Sistemas de workflow refere-se à identificação dos modelos e à análise das ferramentas.

Com o objetivo de investigar e descrever as características dos modelos de sistema workflow, bem como analisar as ferramentas para implantação desse sistema no âmbito das instituições arquivísticas, este estudo apresenta os modelos de workflows existentes e, também, um comparativo entre eles, a fim de identificar o modelo que melhor se ajusta às necessidades das instituições arquivísticas.

Ademais, aponta critérios para coleta e análise de um software de workflow e recomenda critérios para uma ferramenta de workflow que atenda as necessidades arquivísticas, além da análise das ferramentas encontradas.

\section{Revisão da literatura}

\subsection{Gestão de documentos}

Após a II Guerra Mundial, a produção de informação cresceu de uma forma avassaladora, obrigando as instituições a buscarem novas soluções para administrar o grande volume documental acumulado. Nesse meio, além da arquivística e da Teoria das Três Idades, que a norteia, surgiu o conceito de gestão de documentos, conforme Lei Federal:

Considera-se gestão de documentos o conjunto de procedimentos e operações técnicas referentes à sua produção, tramitação, uso, avaliação e arquivamento em fase corrente e intermediária, visando a sua eliminação ou recolhimento para guarda permanente (BRASIL, 1991).

Alguns objetivos da gestão de documentos, conforme Lopes (1996), são: assegurar a produção, administração, manutenção e destinação dos documentos; garantir a acessibilidade da informação quando e onde seja necessária e avaliar a documentação de acordo com seus valores estabelecendo o destino da mesma em tabelas de temporalidade. 
Sob essa ótica, Paes (2005, p. 53) destaca três fases básicas da gestão de documentos: "a produção, a utilização e a destinação". Ainda conforme a autora, a "produção de documentos refere-se à elaboração dos documentos em decorrência das atividades de um órgão ou setor" (PAES, 2005, p. 54).

Nesse contexto, Santos (2005, p. 151), entende que "a produção de um documento é decorrente de uma demanda administrativa ou técnica que precisa ser registrada para que produza efeito e possa servir de comprovação da execução desta demanda". Ainda de acordo com Santos, a produção contempla os procedimentos relacionados à manutenção do maior rigor possível na produção dos documentos de arquivo, abrangendo definição de normas, conteúdo, modelos, formato e trâmite.

\subsection{Tecnologia da Informação}

Somerville (1993 apud FLORES, 2006) afirma que "a Tecnologia da Informação, TI, é o fruto de uma relação transdisciplinar entre a Ciência da Informação e a Ciência da Computação".

Devido ao fato de a TI ter diversas aplicações e estar ligada às mais diversas áreas, torna-se difícil definir e determiná-la por completo. Mas, pode-se afirmar que a Tecnologia da Informação nada mais é do que um conjunto de todas as atividades e soluções providas por recursos de computação.

De acordo com Cruz (1998), o conceito de TI pode ser todo e qualquer dispositivo que tenha capacidade para tratar dados e/ ou informações, tanto de forma sistêmica como esporádica, quer esteja aplicada ao produto, quer esteja aplicada ao projeto. Outro conceito para TI é o que a define como:

Uma gama de produtos de hardware e software que proliferam rapidamente, com capacidade de coletar, armazenar, processar e acessar números e imagens, para o controle dos equipamentos e processos de trabalho e, para conectar pessoas, funções e escritórios, tanto dentro quanto entre as organizações (WALTON, 1994 apud THIVES JÚNIOR, 1999, p. 23).

Sob a mesma ótica, pode-se buscar um conceito mais amplo para a TI, "incluindo os sistemas de informação, o uso de hardware e software, telecomunicações, automação, recursos multimídia, utilizados pelas organizações para fornecer dados, informações e conhecimento" (WEIL, 1992 apud RABECHINI et al., 2001, p. 161).

Segundo Rezende e Abreu (2001), todos esses componentes não teriam utilidade sem o componente fundamental que é o recurso humano, peopleware ou humanware. Embora esse componente não faça parte da Tecnologia da Informação, sem ele essa tecnologia não teria funcionalidade.

\subsection{Workflow}

Segundo Cruz (1998), workflow é a tecnologia que possibilita automatizar processos, racionalizando-os e potencializando-os, por meio de dois componentes implícitos: organização e tecnologia. 
Para o Workflow Management Coalition (WMC, 1996 apud CRUZ, 2001, p. 66), "workflow consiste em um facilitador computadorizado ou automático de um processo de negócio, em parte ou no todo. Sendo assim, pode-se definir workflow como automatização de processos".

Para gerenciar os processos, existem várias tecnologias disponíveis, tais como: workgroup e workflow. Nesse contexto, é relevante diferenciá-las, pois muitos usuários as consideram como mesma tecnologia.

Segundo Cruz (1998), o modelo workgroup é baseado na informação, onde o foco principal está na informação que será processada. Já no workflow, a ênfase é dada ao processo, sendo ele o meio pelo qual a informação será processada.

Há mais um modelo que, de certo modo, se aproxima dos dois abordados, podendo ser considerado como um modelo base, do qual partiram as outras tecnologias. Em resumo, groupware, de acordo com Cruz (2001, p. 33).

É um conjunto de ferramentas que tem por finalidade aumentar a produtividade do trabalho cooperativo, ou seja, é todo e qualquer sistema computadorizado que permita que grupos de pessoas trabalhem de forma cooperativa a fim de atingir um objetivo comum.

Assim, pode-se afirmar que, na essência, o workflow tem muito do groupware, pois permite que um grupo de pessoas trabalhe cooperativamente com um objetivo comum. Porém, o workflow tem como objetivo o processo, orientando o fluxo de documentos e de tarefas, permitindo que várias pessoas trabalhem com o mesmo arquivo ou documento.

Nessa perspectiva, a tecnologia de workflow pode ser aplicada em diversas atividades, lembrando que sempre terá enfoque no processo. Miranda (s. d.) afirma que:

As diferentes estruturas de processos encontradas na prática, fazem com que alguns modelos de workflow apresentem características que os distingue entre si. A identificação dos diferentes tipos permitirá a escolha do modelo mais adequado para representação do processo a ser implantado (MIRANDA; FREITAS; FLORES, s. d., p. 11).

Para tanto, é preciso implementar uma técnica de modelagem que melhor se adapte ao processo. Nesse contexto, as diferentes estruturas de processos encontrados na prática fazem com que alguns modelos de workflow apresentem características que os distinguem entre si. A identificação dos diferentes tipos permitirá a escolha do modelo mais adequado para representação do processo a ser implantado.

\section{Metodologia da pesquisa}

Para o desenvolvimento deste trabalho, realizou-se a revisão da literatura pertinente ao tema de pesquisa escolhido.

Em relação ao sistema workflow, verificou-se que ele apresenta diferentes modelos estruturais diferentes entre si. Desse modo, coube comparar e examinar esses modelos, com o objetivo de buscar identificar o que melhor se ajusta às necessidades das instituições, no que tange ao fazer arquivístico. 
As ferramentas de um Sistema workflow tratam de uma área de pesquisa pouca abordada, não havendo uma bibliografia especializada. Assim, definiu-se critérios para coleta das ferramentas, os quais são: disponibilidade na web, software proprietário/não-proprietário e ferramentas caracterizadas como de Gerenciamento de Documentos (DMS).

Sobre a análise das ferramentas, elaborou-se critérios de acordo com as suas prerrogativas de diagnóstico e classificação. Para indicar a ferramenta de um Sistema workflow que melhor atenda às instituições arquivísticas, levou-se em conta a análise realizada nos softwares apontados neste estudo, bem como os requisitos definidos no trabalho.

\section{Resultados}

\subsection{Exame e identificação do modelo mais adequado às instituições arquivísticas}

De acordo com alguns autores, definiu-se os tipos disponíveis no mercado. Segundo Plesums (2002 apud SANTANA, 2006, p. 5-6), os modelos de workflow podem ser caracterizados de três formas distintas: ad hoc, produção e administrativo. Seguindo a mesma linha, Georgakopopoulos e Hornick (1995 apud THIVES JR., 1999, p. 34) e Kobelius (1995 apud THIVES JR., 1999, p. 34) também apontam para essas três formas. Cruz (1998, p. 86), no entanto, acrescenta os seguintes modelos: orientado para o objeto e baseado no conhecimento.

Já Koulopoulos (1995 apud THIVES JR., 1999, p. 34), aponta quatro métodos de desenvolvimento: "ad hoc, baseado em transações (produção), orientado a objetos e baseado em conhecimento". Ainda conforme o autor, "estes métodos podem ser aplicados em três modelos de processos: centrado em correio eletrônico, centrado em documentos e centrado em processos".

Em relação aos modelos citados acima, o modelo ad hoc caracteriza-se por ser o mais básico de todos e o mais utilizado. É capaz de descrever processos simples, nos quais não há um padrão fixo para o fluxo de informações entre as pessoas envolvidas. Cruz salienta que:

O tipo ad hoc é uma forma eficiente para a racionalização do processo de comunicação dentro da empresa. Em muitos casos, é o primeiro passo para a implantação de tipos mais elaborados da tecnologia (CRUZ, 1998, p. 88).

O workflow do tipo produção (transação) é pré-definido e priorizado, suportando assim um grande volume informacional. De acordo com Koch (1998, p. 42-43):

O Workflow de produção gerencia fluxos de trabalho e a integração de ferramentas em processos estruturados, possuindo interface gráfica para 0 desenho do fluxo e mecanismos sofisticados de controle de processos que envolvam altos valores e volumes, como por exemplo, no mercado nacional, os processos de concessão de crédito, câmbio e sinistro em seguros. 
Já o modelo administrativo é um meio-termo entre um workflow "ad hoc" e um de "produção", ou seja, envolve atividades fracamente estruturadas, repetitivas, previsíveis e com regras simples de coordenação de tarefa.

Para Cruz (1998, p. 91-92), "o workflow orientado a objeto constitui uma versão mais sofisticada do orientado a transação". O autor ainda ressalta que a teoria Orientação a Objetos não surgiu com a tecnologia workflow, mas sim na década de 1980, com uma "evolução da tecnologia reacional", destinada a bancos de dados.

Com base no entendimento de Thives Jr. (1999, p. 36), pode-se afirmar que o workflow baseado em conhecimento tem características e ferramentas que permitem aprender com os erros e acertos advindos da implantação deste. Assim sendo, Koulopoulos (1995 apud THIVES JR., 1999, p. 36) afirma que as tecnologias-base nesse tipo são a da inteligência artificial e a dos sistemas especialistas, as quais possibilitam aos Sistemas de workflow aprender por si próprios e inferir soluções, a partir da vivência de fatos do cotidiano.

Com a identificação dos modelos de workflow existentes e de acordo com as características apontadas por cada modelo, pode-se apontar o modelo que melhor se ajusta às necessidades das instituições arquivísticas.

Assim, tem-se que o modelo ad hoc é o mais indicado para suprir as necessidades de instituições que buscam gerir as informações arquivísticas de uma forma eficiente, possibilitando dessa forma, a racionalização do processo de comunicação dentro das empresas. Nesse contexto, Koulopoulos (1997 apud THIVES JR., 1999, p. 36), defende que "a característica de racionalizar a comunicação dentro da instituição é o primeiro passo para a implantação de tipos mais elaborados desta tecnologia".

Desse modo, fica evidente que ao definir o modelo ad hoc, levou-se em conta o fato de que esse sistema de workflow é o mais elementar que existe, tornando-se, dessa forma, o mais abrangente, pois, de acordo com Thives Jr. (1999, p. 37), "é utilizado em processos onde não há um padrão pré-estabelecido de fluxo/trâmite da informação entre as pessoas".

No entanto, apesar do sistema ad hoc ser o mais simples, requer a mesma infra-estrutura tecnológica que os demais. Sua implantação requer uma mudança cultural na organização nem sempre pequena e nem sempre simples, como afirma Cruz (1998). Porém, dos sistemas disponíveis atualmente, ele ainda é o mais eficiente no que diz respeito à implementação e, consequentemente, à quebra de resistência por parte dos usuários.

Com o modelo de workflow definido, faz-se necessário identificar quais as ferramentas disponíveis para sua implementação. Assim, deve-se definir os critérios de análise dos softwares de workflow.

\subsection{Definição dos critérios para levantamento e análise dos softwares}

Pode-se definir que um dos critérios da análise de softwares é a sua disponibilidade na web, pois é a forma mais ágil e rápida de se ter acesso a eles. Outro critério identificado na pesquisa foi a versatilidade das ferramentas 
existentes, ou seja, elas necessitam rodar em sistemas operacionais proprietários, como é caso do Windows e também em sistemas não proprietários, como o Linux.

Assim sendo, foi possível listar uma gama de ferramentas que atendiam a esses critérios e com as suas descrições, arrolou-se alguns dados sobre esse levantamento, conforme QUADRO 1.

\section{QUADRO 1}

Listagem das ferramentas encontradas

\begin{tabular}{|c|c|}
\hline Características das ferramentas & Ferramentas \\
\hline $\begin{array}{l}\text { Ferramentas caracterizadas por serem Sistemas } \\
\text { de Gerenciamento de Conteúdo (CMS) }\end{array}$ & $\begin{array}{l}\text { Joomla } \\
\text { Mambo } \\
\text { Plone } \\
\text { Drupal }\end{array}$ \\
\hline $\begin{array}{l}\text { Ferramentas caracterizadas por serem Sistemas } \\
\text { Gerenciadores de Documentos (DMS) }\end{array}$ & $\begin{array}{l}\text { Alfresco } \\
\text { Agorum Core } \\
\text { KnowledgeTree } \\
\text { ArchivistaBox } \\
\text { Maarch } \\
\text { Owl Intranet }\end{array}$ \\
\hline $\begin{array}{l}\text { Ferramenta caracterizada por ser um Sistema de } \\
\text { controle de informações descritivas }\end{array}$ & ICA-ATOM \\
\hline
\end{tabular}

Fonte: LAMPERT (2009, p. 52).

Em virtude de se ter encontrado uma diversidade de ferramentas que atendam às especificações já estabelecidas e de acordo com os dados apontados no QUADRO 1, fez-se necessário refinar o resultado encontrado. Desse modo, definiu-se um novo critério para análise das ferramentas.

Por se tratar de uma pesquisa embasada nos preceitos arquivísticos, o critério utilizado foi analisar apenas as ferramentas criadas para Gerenciamento de Documentos (DMS), visto que o profissional arquivista deve, dentre as demais funções, participar da produção dos documentos eletrônicos, cooperando na concepção e no desenvolvimento de sistemas automatizados de informação (RABECHINI et al., 2001).

Logo, restaram as seguintes ferramentas: Agorum Core, Alfresco, ArchivistaBox, KnowledgeTree, Maarch e Owl Intranet.

Definidas as ferramentas, apontou-se outros critérios para analisá-las, uma vez que os critérios abordados anteriormente eram para refinar os resultados do levantamento realizado e a primeira amostragem apontou um grande número de ferramentas.

Nesse contexto, definiu-se alguns critérios de acordo com as prerrogativas de diagnóstico e classificação das ferramentas, que são: ferramentas contendo um Sistema de workflow; tempo de download (tamanho do arquivo); prérequisitos para instalação; compatibilidade com o Sistema Operacional; necessidade do conhecimento de uma língua estrangeira para operar o software; opção on-line para testar a ferramenta; número de Sistemas Operacionais disponíveis; realização de webinar $^{1}$ para treinamento; e divulgação das funcionalidades do software.

\footnotetext{
1 Webinar é uma abreviação para "web-based seminar", ou seja, trata-se simplesmente de um seminário realizado em ambiente web. Pode-se definir também, como uma conferência on-line.
} 


\subsection{Recomendação de requisitos para uma ferramenta de workflow adequada às necessidades arquivísticas}

Para recomendar requisitos de uma ferramenta de workflow adaptada às necessidades arquivísticas, deve-se levar em conta o e-ARQ Brasil, que estabelece um modelo de requisitos para um Sistema Informatizado de Gestão Arquivística de Documentos (SIGAD).

O e-ARQ Brasil (2006) está estruturado em duas partes. A primeira dando ênfase às teorias e conceitos que norteiam a Gestão Arquivística de Documentos. A segunda, destacando a especificação de requisitos para o SIGAD e apresentando aspectos de funcionalidade de um sistema informatizado, dentre as quais destacam-se a Tramitação e Fluxo de Trabalho.

Nesse contexto, pode-se afirmar que o e-ARQ Brasil (2006, p. 56) apresenta os "requisitos de uma ferramenta de workflow adaptada às necessidades arquivísticas", de acordo com o capítulo que compreende Tramitação e Fluxo de Trabalho. Esse capítulo aponta requisitos dos casos em que um SIGAD inclui recursos de automação de fluxo de trabalho (workflow), isto é, abrange funções para controle do fluxo de trabalho e atribuição de metadados para registro da tramitação dos documentos, incluindo o status do documento (minuta, original ou cópia).

Assim sendo, conforme o e-ARQ Brasil (e-ARQ, 2006, p. 56), os recursos de um SIGAD para controle do fluxo de trabalho podem compreender: tramitação de um documento antes do seu registro/captura; e tramitação posterior ao seu registro/captura.

Ainda conforme o SIGAD, as tecnologias de fluxo de trabalho transferem objetos digitais entre participantes, sob o controle automatizado de um programa. São geralmente usadas para:

a) gestão de processos ou de tarefas, tais como registro e destinação de documentos e dossiês/processos;

b) verificação e aprovação de documentos ou dossiês/processos antes do registro;

c) encaminhamento de documentos ou dossiês/processos de forma controlada, de um usuário para outro , com a identificação das ações a serem realizadas tais como: "verificar documento" e "aprovar nova versão";

d) comunicação aos usuários sobre a disponibilidade de um documento arquivístico;

e) distribuição de documentos ou dossiês/processos; e

f) publicação de documentos ou dossiês/processos na web.

\subsection{Análise e indicação das ferramentas que melhor atendem às instituições arquivísticas}

Definida a listagem das ferramentas, chegando-se a um total de seis, em um primeiro momento, pode-se realizar a análise do conjunto e, posteriormente, fez-se a análise de cada uma delas. 
Desse modo, foi possível identificar algumas semelhanças, tais como: todas as ferramentas relacionadas são Open Source (Software livre); todos os softwares disponibilizam opção para download em seus websites; para utilizar os softwares e navegar nos respectivos websites necessita-se de familiaridade com alguma língua estrangeira; a maior parte das ferramentas possui em sua página da web a opção de teste on-line; a maioria das ferramentas disponibiliza os softwares nos Sistemas Operacionais (SO) Windows, Linux e Mac; todas as ferramentas necessitam para instalação ao menos um desses pré-requisitos:

a) um servidor web livre, sendo o Apache2 o mais utilizado;

b) um SGBD (Sistema Gerenciador de Banco de Dados). O mais empregado é o MySQL3;

c) uma linguagem de computadores para gerar conteúdo dinâmico na World Wibe Web denominada PHP4.

Em virtude dos pré-requisitos apontados, buscou-se uma ferramenta, em portais de pesquisa da internet, para instalar os programas acima. Como resultado dessa pesquisa, chegou-se a softwares denominados WAMP, isto é, um programa que efetua a instalação automática dos softwares Apache, MySQL e PHP para Windows. Deve-se destacar que foram encontrados alguns softwares denominados $L A M P$, ou seja, tem a mesma definição do WAMP, porém são destinados para o SO Linux.

Cabe ressaltar que a instalação dos programas analisados deu-se no Sistema Operacional Windows, distribuição XP Home Professional, uma vez que o pesquisador não é familiarizado com o SO Linux.

\subsubsection{Agorum Core ${ }^{5}$}

É um Sistema de Gestão Documental (DMS) Open Source desenvolvido pela empresa Alemã Agorum Software $\mathrm{GmbH}$. Disponibiliza duas versões: Agorum Core OS e Agorum Core Pro.

A versão Agorum Core OS é a opção de código aberto sob a licença GNU/GLP. Contém as funções básicas do software, bem como, as mais importantes segundo o website da empresa. Possui duas opções para download nos Sistemas Operacionais Windows e Linux:

a) Agorum Core Server - disponível em Windows (310MB) e Linux (330MB);

b) Agorum Core Client - disponível em Windows (32MB).

A versão Agorum Core Pro oferece todas as funcionalidades do software. Elas estão estruturadas em módulos, tais como: workflow, Share, Arquivo e-mail,

\footnotetext{
${ }^{2}$ Apache é um servidor web livre compatível com o protocolo HTTP. Criado em 1995, é a principal tecnologia da Apache Software Foundation.

${ }^{3}$ MySQL é um Software Livre com base na GLP, que utiliza como interface a linguagem SQL.

${ }^{4}$ PHP é uma linguagem de programação de computadores interpretada, livre e amplamente utilizada para gerar conteúdo ma Web.

${ }^{5}$ Agorum Software GmbH. Disponível em: <http://www.agorum.com>. Acesso em: 06 out. 2009.
} 
Metadados e outros. Porém, essa versão não está disponível para download, uma vez que está sob licença, com o código fechado.

Desse modo, não foi possível testar e analisar o Sistema de workflow do Agorum Core na prática, visto que essa funcionalidade só está disponível na versão Pro. No entanto, o website da ferramenta aponta algumas características e propriedades do módulo workflow:

a) atribuir tarefas para indivíduos ou para grupos;

b) vincular documentos referente ao fluxo de trabalho;

c) data-limite para ação (revisão/aprovação); e

d) edição do workflow durante o processamento do fluxo - caso uma tarefa não tenha sido realizada possua data-limite, pode-se repassá-la para outro grupo ou indivíduo.

\subsubsection{Alfresco ${ }^{6}$}

Caracteriza-se por ser um Sistema Gerenciador de Documentos que possui três funcionalidades principais: Gestão Documental (DMS), Gestão de Conteúdo Web (CMS) e Colaboração (Share).

O Alfresco possui a opção de teste on-line. Assim, o primeiro método utilizado para analisar o programa foi esse, porém, a opção de test-drive do software disponibiliza apenas a funcionalidade de Colaboração. Logo, ao analisar o Alfresco Share, percebeu-se que ele não dispõe de um Sistema de workflow. Portanto, fez-se necessário realizar o download da funcionalidade Gestão Documental, para, em seguida, instalá-lo.

A funcionalidade Gestão Documental disponibiliza duas opções para download, ambas as versões estão disponíveis sob a licença trial $^{7}$ :

a) Alfresco Enterprise Edition - disponível em Windows (310MB), Linux (117MB) e Mac (276MB); e

b) Alfresco Community Edition - disponível em Windows (362MB), Linux (143MB) e Mac (301MB).

Em relação aos tipos básicos do fluxo de trabalho, essa ferramenta apresenta:

a) Workflow simples - basicamente pré-configurado. É uma regra especial que aplica gerenciamento automático ao ciclo de vida do documento. Essencialmente, move ou copia conteúdo baseado em uma ação do usuário;

\footnotetext{
${ }^{6}$ The open source alternative for enterprise content management. Disponível em:<http://www.alfresco.com $>$. Acesso em: 03 out. 2009.

${ }^{7}$ Trial é uma licença de uso de software semelhante ao demo e ao shareware, porém, com limitação de tempo.
} 
b) Workflow avançado - além de contar com as mesmas funções do workflow simples, esse tipo permite diversas configurações do fluxo de trabalho, tais como:

- revisor para o documento e a quantidade de revisores;

- aprovador do documento e quem tem a prioridade na revisão; e

- se há data limite para ação ou qual a data-limite para ação.

\subsubsection{ArchivistaBox ${ }^{8}$}

É um Sistema de Gestão Documental (DMS) Open Source desenvolvido pela empresa alemã Archivista $\mathrm{GmbH}$. Possui integração com um Sistema Integrado de Gestão Empresarial (ERP).

Essa ferramenta possui a opção de teste on-line. Logo, buscou-se analisá-la através dessa funcionalidade. Assim, ao iniciar a versão on-line é possível gerenciar todos os recursos disponíveis no programa, tais como: visualizar imagem/arquivo; servidor PDF; conversão de imagem/arquivo para PDF pesquisável; busca/pesquisa; digitalização; impressão; e outros.

Apesar da versão on-line disponibilizar diversas funcionalidades, não apresenta um Sistema de workflow. Dessa forma, buscou-se fazer o download do software, uma vez que nas demais ferramentas essa versão caracteriza-se por ser mais completa.

Desse modo, após baixar, testar e analisar a versão open source (licença GPLv2) em formato ISO $^{9}$ (978MB), conclui-se que ela também não apresenta um Sistema de workflow.

A ferramenta caracteriza-se, predominantemente, por permitir a gestão de arquivos digitalizados, focando todas as etapas da digitalização de documentos, desde a captura até o armazenamento, preservação dos arquivos digitais e consequente recuperação. Embora seja um programa que não apresenta um Sistema de workflow, deve-se, assim mesmo, apontar algumas funcionalidades dele:

a) número ilimitado de bancos de dados;

b) OCR automático;

c) upload e conversão dados;

d) download de todos os documentos em PDF;

e) upload de imagens de todas as câmeras digitais padrão; e

f) backup executado automaticamente.

\subsubsection{KnowledgeTree ${ }^{10}$}

Conforme o website, trata-se de um software de Gestão Documental (DMS) open source capaz de ligar pessoas, ideias e processos.

\footnotetext{
${ }^{8}$ Open source DMS, ERP \& Virtualization Out-of-the-box. Disponível em: $<$ http://www.archivista.ch/en $>$. Acesso em: 07 out. 2009.

${ }^{9}$ ISO é um dos formatos mais utilizados para gerar um arquivo de imagem (cópia literal de um CD/DVD).

${ }^{10}$ Open source Document Management System. Disponível em:<http://www.knowledgetree.com/pt $>$. Acesso em: 11 out. 2009.
} 
No que diz respeito à análise do software, o método empregado para examinar o programa foi a opção de teste on-line. Nesse teste é possível gerenciar as funcionalidades disponíveis no programa, tais como: explorar documentos, preferências, busca/pesquisa, nuvem de etiquetas (metadados), administração e outras.

Após analisar as funcionalidades da opção de teste on-line, deve-se apontar alguns recursos das versões disponibilizadas para download, uma vez que elas podem conter mais funções no que tange aos Sistemas de workflow.

Assim sendo, a ferramenta open source (licença GPL v3) possui duas opções de download, ambas as versões disponíveis sob a licença trial:

KnowledgeTree 3,7 - disponível em Windows (378MB) e Linux (3MB);

KnowledgeTree Community Edition - disponível em Windows (59MB) e Linux (56MB). Essa opção permite instalar a partir do código-fonte, porém necessita dos aplicativos Apache, PHP e MySQL instalados.

A versão KnowledgeTree 3,7 caracteriza-se por ser voltada às pessoas, ideias e processos. Já a versão KnowledgeTree Community Edition permite sua instalação através do código-fonte. No que diz respeito aos recursos de Workflow da versão KnowledgeTree 3,7, destacam-se:

a) grupo de atribuição de tarefas;

b) estados baseados em fluxo de trabalho;

c) notificações de workflow via e-mail;

d) roteamento de workflow usando restrições de transição;

e) controle de fluxos de trabalho;

f) revisão/aprovação de workflows;

g) fluxo de trabalho automático;

h) iniciar fluxos de trabalho para ações de documentos e pastas.

\subsubsection{Maarch $^{11}$}

É um Sistema de Gestão Documental (DMS) open source desenvolvido pela empresa francesa Maarch SAS. De acordo com seu website, caracteriza-se por ser um conjunto de ferramentas e soluções para gestão e arquivamento de documentos. Conta com duas aplicações operacionais básicas:

a) Maarch LetterBox - possibilita a gestão de correio eletrônico; e

b) Maarch Entreprise - permite a gestão completa dos documentos de uma instituição.

A versão Maarch Enterprise é composta por módulos funcionais que agregam serviços, tais como: gestão de arquivos físicos; gestão de documentos; exportação de arquivos em dispositivos ópticos; digitalização; importação de arquivos; workflow; e outros. Eles podem ser ativados ou desativados para

\footnotetext{
${ }^{11}$ The first open source archiving DMS. Disponível em: <http://www.maarch.org/en>. Acesso em: 05 out. 2009.
} 
atender às necessidades dos usuários. Estão disponíveis para download (licença GPLv3) nos Sistemas Operacionais Windows, Linux e Mac.

Em relação à análise da ferramenta, em um primeiro momento, realizou-se através da opção de teste on-line. Contudo, pelo fato de não possuir o módulo de workflow, procurou-se realizar o download da versão Maarch Enterprise. Após testar a versão, percebeu-se que a mesma não dispunha do módulo de fluxo documental.

Assim sendo, a análise da ferramenta deu-se através do seu website, visto que ele possui um exemplo de workflow para aprovação de faturas contábeis. Baseado nesse exemplo, pode-se notar que o Sistema de workflow da ferramenta tem as mesmas características dos demais, pois possibilita tramitar documentos, tem a função de validação/aprovação e o recurso de informar o envio do trâmite. Contudo, não possui a opção de estipular data-limite para o mesmo.

A ferramenta Maarch destaca-se por respeitar a Norma ISO 15489 (Norma para Gestão de Arquivos) e ao padrão Dublin Core - esquema de metadados.

\subsubsection{Owl Intranet ${ }^{12}$}

É um Sistema de Gestão Documental (DMS), porém, tem como principal aplicação um repositório de documentos multi-usuário (knowledgebase) escrito em PHP. De acordo com seu website, é voltado para a publicação de arquivos e documentos web em grandes e pequenas empresas, bem como em corporações on-line.

Essa ferramenta possui a opção de teste on-line e, por essa razão, buscouse analisá-la através dessa funcionalidade. Os recursos disponíveis nessa versão são: gestão de e-mail, busca de arquivos, criação de tipos documentais e outros.

Apesar de possuir uma gama de opções para gestão documental, a versão de teste on-line não apresenta um Sistema de workflow. Assim, buscou-se pesquisar no website da ferramenta os recursos, para verificar se há um sistema de fluxo de trabalho. Logo, foi possível identificar as principais características e funcionalidades do software, dispostas a seguir:

a) notificação e permissão para grupo de usuários;

b) ferramenta de pesquisa integrada em diversos formatos;

c) capacidade para armazenar arquivos no sistema ou em banco de dados;

d) ferramenta para gestão de e-mail;

e) upload com extração automática de arquivos ZIP;

f) download de arquivos em formato PDF.

Após identificar as funcionalidades e verificar as opções para download no que tange ao fluxo de trabalho, conclui-se que a ferramenta não possui um Sistema de workflow. Caracteriza-se, predominantemente, por ser um repositório de documentos multi-usuário (knowledgebase).

Em relação à indicação da ferramenta, elaborou-se um quadro para melhor entendimento das características e averiguação de atendimento aos critérios definidos. Logo, tem-se o QUADRO 2, disposto a seguir:

${ }^{12}$ Owl Intranet Engine. Disponível em: <http://www.owl.anytimecomm.com>. Acesso em: 05 out. 2009. 


\section{QUADRO 2}

Critérios para análise das ferramentas

\begin{tabular}{|c|c|c|c|c|c|c|}
\hline $\begin{array}{l}\text { FERRAMENTAS } \\
\text { CRITÉRIOS }\end{array}$ & 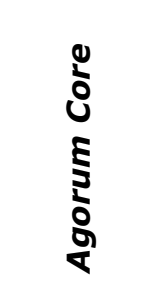 & $\begin{array}{l}8 \\
y \\
\frac{5}{4} \\
\frac{5}{4}\end{array}$ & 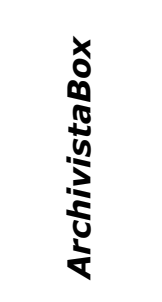 & 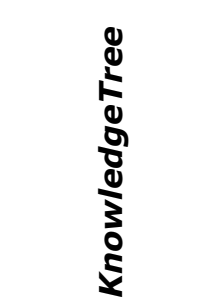 & $\frac{5}{\frac{1}{0}}$ & 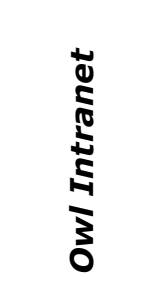 \\
\hline Contém Sistema workflow & Sim & Sim & Não & Sim & Sim & Não \\
\hline $\begin{array}{l}\text { Tempo de download } \\
\text { (tamanho do arquivo) }\end{array}$ & $300 \mathrm{MB}$ & $300 \mathrm{MB}$ & $970 \mathrm{MB}$ & $370 \mathrm{MB}$ & $5 M B$ & $30 \mathrm{MB}$ \\
\hline $\begin{array}{l}\text { Necessidade de Língua } \\
\text { Estrangeira }\end{array}$ & Alemão & $\begin{array}{l}\text { Não há } \\
\text { necessidade }\end{array}$ & $\begin{array}{l}\text { Alemão } \\
\text { ou } \\
\text { Inglês }\end{array}$ & $\begin{array}{l}\text { Não há } \\
\text { necessidade }\end{array}$ & $\begin{array}{c}\text { Francês } \\
\text { ou } \\
\text { Inglês }\end{array}$ & Inglês \\
\hline Opção de teste on-line & Não & Sim & Sim & Sim & Sim & Sim \\
\hline $\begin{array}{l}\text { Sistemas Operacionais } \\
\text { disponíveis }\end{array}$ & $\begin{array}{l}\text { Windows } \\
\text { Linux }\end{array}$ & $\begin{array}{c}\text { Windows } \\
\text { Linux } \\
\text { Mac }\end{array}$ & $\begin{array}{l}\text { Windows } \\
\text { Linux }\end{array}$ & $\begin{array}{c}\text { Windows } \\
\text { Linux } \\
\text { Mac }\end{array}$ & $\begin{array}{c}\text { Windows } \\
\text { Linux } \\
\text { Mac }\end{array}$ & $\begin{array}{l}\text { Windows } \\
\text { Linux }\end{array}$ \\
\hline $\begin{array}{l}\text { Compatibilidade com o } \\
\text { Sistema Operacional }\end{array}$ & Sim & Sim & Sim & Sim & Sim & Sim \\
\hline $\begin{array}{l}\text { Realização e divulgação de } \\
\text { webinar }\end{array}$ & Não & Sim & Não & $\operatorname{Sim}$ & Não & Sim \\
\hline $\begin{array}{l}\text { Pré-requisitos para } \\
\text { instalação }\end{array}$ & $\begin{array}{c}\text { Apache } \\
\text { MySQL } \\
\text { PHP }\end{array}$ & $\begin{array}{l}\text { Apache } \\
\text { MySQL }\end{array}$ & $\begin{array}{c}\text { MySQL } \\
\text { PHP } \\
\text { Perl }\end{array}$ & $\begin{array}{c}\text { Apache } \\
\text { MySQL } \\
\text { PHP }\end{array}$ & $\begin{array}{c}\text { Apache } \\
\text { MySQL } \\
P H P\end{array}$ & $\begin{array}{c}\text { Apache } \\
\text { MySQL } \\
\text { PHP }\end{array}$ \\
\hline
\end{tabular}

Fonte: LAMPERT (2009, p. 88).

Um critério que não foi abordado durante o estudo, mas deve ser apontado para identificar a melhor ferramenta, diz respeito a sua facilidade de instalação. Logo, o software de instalação mais fácil foi o KnowledgeTree, visto que conta com um programa integrado para auxiliar a instalação. Ademais, apresenta em seu website um tutorial para essa tarefa. Cabe destacar que as ferramentas Agorum Core, Alfresco e Maarch contam com tutoriais e/ou vídeos para auxiliar a instalação.

Assim, com base nas informações expostas no QUADRO 2 e de acordo com as impressões do pesquisador, pode-se apontar as ferramentas Alfresco e KnowledgeTree como aquelas que melhor atendem às instituições arquivísticas. A seguir, pela ordem estão Maarch, Agorum Core, ArchivistaBox e Owl Intranet.

Por fim, é relevante destacar que as ferramentas analisadas são constantemente atualizadas por novas versões, estando em constante evolução e aprimoramento. Assim, as considerações apresentadas sobre cada software são aplicáveis aos atuais estágios de desenvolvimento e, certamente, em um futuro próximo, serão desatualizadas por novas versões. Logo, pode-se afirmar que as ferramentas são altamente dinâmicas. 


\section{Considerações finais}

O presente artigo apresentou a tecnologia de workflow sob a ótica da arquivística, identificando os sistemas de workflow, os modelos existentes e a análise das ferramentas definidas pelos critérios da pesquisa.

Com a popularização da informática e a expansão da tecnologia da informação dentro das instituições, o volume de processos e tarefas aumentou vertiginosamente nos últimos anos. Assim sendo, os profissionais da informação buscaram otimizar os fluxos de informação e os processos organizacionais através da tecnologia da informação. O resultado foi a tecnologia do workflow.

Nesse contexto, com base nas diversas teorias abordadas durante a realização deste estudo, bem como nos dados obtidos com a análise das ferramentas de um Sistema workflow, pode-se identificar as características dos modelos e as funcionalidades dos softwares analisados.

Assim, foi possível identificar uma gama de ferramentas que atendem aos critérios propostos pelo trabalho, que são: Joomla; Mambo; Plone; Drupal (identificadas como Sistemas de Gerenciamento de Conteúdo, CMS); ICA-ATOM (identificada como Sistema de Controle de Informações Descritivas). No entanto, apenas as ferramentas Agorum, Alfresco, ArchivistaBox, KnowledgeTree, Maarch e Owl Intranet foram analisadas por caracterizarem-se como Sistemas de Gestão Documental(DMS).

Desse modo, os resultados da pesquisa apontaram o modelo de workflow mais adequado às instituições arquivísticas, os requisitos de uma ferramenta de workflow para as necessidades arquivísticas e as ferramentas que melhor atendem às instituições arquivísticas. Portanto, as ferramentas Alfresco e KnowledgeTree apresentaram-se como as mais qualificadas, de acordo com as prerrogativas de diagnóstico, classificação das ferramentas e averiguação de atendimento aos critérios definidos.

Ao fim do estudo, pode-se identificar algumas semelhanças das ferramentas, tais como: o caráter dinâmico que elas possuem, visto que estão em constante aprimoramento e a acessibilidade por navegador ou browser (webbased), possuindo estrutura Internet "cliente-servidor".

\section{Referências}

BRASIL. Presidência da República Federativa do Brasil. Lei n. 8.1589, de 8 de janeiro de 1991 . Dispõe sobre a política nacional de arquivos públicos e privados e dá outras providências. Disponível em: <http://www.planalto.gov.br/ccivil 03/LEIS/L8159.htm>. Acesso em: 18 ago. 2008.

CONSELHO NACIONAL DE ARQUIVOS (CONARQ). e-ARQ Brasil: modelo de requisitos para sistemas informatizados de gestão arquivística de documentos. Câmara Técnica de Documentos Eletrônicos - CTDE, 2006. Disponível em <http://www.conarq.arquivonacional.gov.br/Media/publicacoes/earqbrasilv1.pdf> . Acesso em: 02 jun. 2008.

CRUZ, T. e-Workflow: como implantar e aumentar a produtividade de qualquer processo. São Paulo: CENADEM, 2001. 
1998.

. Workflow: a tecnologia que vai revolucionar processos. São Paulo: Atlas,

FLORES, D. A gestão eletrônica de documentos (GED) e o impacto das políticas de software livre: uma perspectiva transdisciplinar, comparada nos arquivos do Brasil e Espanha. 2006. Tese (Doutorado em Metodologías y Líneas de Investigación en Biblioteconomía y Documentación) - Universidad de Salamanca/Espanha, 2006.

GEORGAKOPOPOULOS, D.; HORNICK, M. An overview of workflow management: from process modeling to workflow automation infrastructure. Distributed and Parallel Databases, n. 3, p. 119-153, 1995 apud THIVES, J. J. J. Workflow, uma tecnologia para transformação do conhecimento nas organizações. 1999. Dissertação (Mestrado em Administração) - Universidade Federal de Santa Catarina, Florianópolis, 1999.

KOBELIUS, J. The rhytm of work: a buyer's guide to workflow tools. Network World/Collaboration, November/December, 1995 apud THIVES, J. J. J. Workflow, uma tecnologia para transformação do conhecimento nas organizações. 1999. Dissertação (Mestrado em Administração) - Universidade Federal de Santa Catarina, Florianópolis, 1999.

KOCH, W. W. Gerenciamento eletrônico de documentos - GED: conceitos, tecnologias e considerações finais. São Paulo: CENADEM, 1998.

KOULOPOULOS, T. M. The workflow imperative: building real work business solutions. U.S.A: John Wiley \& Sons, 1995 apud THIVES, J. J. J. Workflow, uma tecnologia para transformação do conhecimento nas organizações. 1999. Dissertação (Mestrado em Administração) - Universidade Federal de Santa Catarina, Florianópolis, 1999.

Creating the knowing enterprise: the evolution of knowledge management. U.S.A: KMWord White Paper, 1997 apud THIVES, J. J. J. Workflow, uma tecnologia para transformação do conhecimento nas organizações. 1999. Dissertação (Mestrado em Administração) - Universidade Federal de Santa Catarina, Florianópolis, 1999.

LAMPERT, S. R. Os sistemas de workflow em arquivística: a identificação dos modelos e a análise das ferramentas. Monografia (Graduação em Arquivologia) Universidade Federal de Santa Maria, Santa Maria, 2010.

LOPES, L. C. A informação e os arquivos: teorias e práticas. Niterói: EDUFF; São Carlos: EDUFSCar, 1996.

MIRANDA, R. S.; FREITAS, L. A. R.; FLORES, D. Sistema de workflow: um estudo de implantação. s.d. Disponível em: <http://www.caarq.com.br/textoseartigos.html>. Acesso em: 25 jun. 2008.

PAES, M. L. Arquivo: teoria e prática. 5 reimp. Rio de Janeiro: FGV, 2005.

PLESUMS, C. The workflow handbook 2002: introduction to workflow. Florida: Future Strategies Inc., 2002 apud SANTANA, J. W. S. Sistemas workflow: uma aplicação ao IC. 2006. Monografia (Graduação em Ciência da Computação) Universidade Federal de Alagoas, Maceió, 2006. Disponível em: 
<http://www.bani.com.br/wp-content/uploads/2006/07/tcc Wendell.pdf > . Acesso em: 06 set. 2008.

RABECHINI, R. Jr. et al. O papel da tecnologia da informação (TI) na estratégia das organizações. Gestão e Produção, São Carlos, v. 8, n. 2, p. 160-179, ago 2001. Disponível em: <http://www.scielo.br/pdf/gp/v8n2/v8n2a04.pdf>. Acesso em: 12 de out. 2009.

REZENDE, D. A.; ABREU, A. F. Tecnologia da informação aplicada a sistemas de informação empresarias. 2 ed. São Paulo: Atlas, 2001.

SANTANA, J. W. S. Sistemas workflow: uma aplicação ao IC. 2006. Monografia (Graduação em Ciência da Computação) - Universidade Federal de Alagoas, Maceió, 2006. Disponível em: <http://www.bani.com.br/wpcontent/uploads/2006/07/tcc Wendell.pdf>. Acesso em: 06 set. 2008.

SANTOS, V. B. Gestão de documentos eletrônicos: uma visão arquivística. 2 ed. Ver. Aum. Brasília: ABARQ, 2005.

SOMERVILLE, M. A. Transdisciplinaridade, onda do futuro: como preparar nossas praias. Revista Tempo Brasileiro. Rio de Janeiro, v.113, p. 75-96, abr./jun., 1993 apud FLORES, D. A gestão eletrônica de documentos (GED) e o impacto das políticas de software livre: uma perspectiva transdisciplinar, comparada nos arquivos do Brasil e Espanha. 2006. Tese (Doutorado em Metodologías y Líneas de Investigación en Biblioteconomía y Documentación) - Universidad de Salamanca/Espanha, 2006.

THIVES, J. J. J. Workflow, uma tecnologia para transformação do conhecimento nas organizações. 1999. Dissertação (Mestrado em Administração) - Universidade Federal de Santa Catarina, Florianópolis, 1999.

WALTON, R. E. Tecnologia de informação: o uso da TI pelas empresas que obtém vantagem competitiva. São Paulo: Atlas, 1994 apud THIVES, J. J. J. Workflow, uma tecnologia para transformação do conhecimento nas organizações. 1999. Dissertação (Mestrado em Administração) - Universidade Federal de Santa Catarina, Florianópolis, 1999.

WEIL, P. The relationship between investmen in information technology and firm performance: a study of the valve manufcturing sector. Information Systems Research, v. 3, n. 4, p. 307-333, 1992 apud RABECHINI, R. Jr. et al. O papel da tecnologia da informação (TI) na estratégia das organizações. Gestão e Produção, São Carlos, v. 8, n. 2, p. 160-179, ago 2001. Disponível em: $<$ http://www.scielo.br/pdf/gp/v8n2/v8n2a04.pdf>. Acesso em: 12 de out. 2009.

WORKFLOW MANAGEMENT COALITON. Terminology \& Glossary. Belgium: [s.n.], 1996 apud CRUZ, T. e-Workflow: como implantar e aumentar a produtividade de qualquer processo. São Paulo: CENADEM, 2001. 